\title{
Tres décadas de muy baja fecundidad en España, 1991 - 2018
}

\author{
Albert Esteve*, Mariona Lozano*, Diederik Boertien*, Ryohei Mogi*, Qi Cui* \\ * Centre d'Estudis Demogràfics / CERCA Programme, Universitat Autònoma de Barcelona
}

\section{Resumen}

Con datos de la Encuesta de Fecundidad de 2018 (14.556 mujeres y 2.619 hombres), en este artículo (i) examinamos la evolución de la fecundidad de las generaciones de mujeres y hombres nacidos en España entre 1962 y 1989 y sus preferencias reproductivas en cuanto al tamaño ideal de familia y al deseo de tener hijos en los próximos tres años; (ii) analizamos los motivos por los que mujeres y hombres no han alcanzado el número deseado de hijos y estimamos la fecundidad no realizada según sea el motivo principal que la ha impedido; $y$, finalmente, (iii) examinamos la probabilidad de tener un primer hijo en función de la edad y la situación de pareja y laboral de las personas. Los resultados constatan el carácter estructural de la baja fecundidad en España para mujeres y hombres. La fecundidad observada está claramente por debajo de la fecundidad deseada. Los motivos materiales - asociados con la precariedad laboral, la inestabilidad, los recursos económicos - son los principales responsables de que mujeres y hombres no alcancen el número de hijos deseados. Tras estos motivos aparecen los relacionados con la pareja (no tener pareja estable) y la salud (dificultades de quedar embarazada). Tener pareja estable entre los 25 y 35 años es un determinante próximo de la transición al primer hijo. 


\section{Introducción}

En 1991, España fue, junto a Italia, el primer país del mundo en registrar una fecundidad por debajo de los 1.3 hijos por mujer (Kohler, et al., 2002). Tres décadas más tarde, la fecundidad seguía por debajo de este umbral. Según las estimaciones del Instituto Nacional de Estadística (INE), la fecundidad registrada en 2019 fue de 1,23 hijos para todas las mujeres y de 1,17 para las nacidas en España. Por cohorte de nacimiento, ninguna generación de mujeres nacida en España desde 1960 ha alcanzado los 1,4 hijos por mujer al cumplir los 40 años. A pesar de estas cifras, que sitúan a España como uno de los países más envejecidos del mundo (Naciones Unidas, 2019), las causas de su baja fecundidad han sido poco estudiadas debido, principalmente, a la escasez de datos. La realización de encuestas de fecundidad ha sido parca e irregular en el tiempo y no ha habido participación española en el programa de la "Generations and Gender"; cuyas encuestas han nutrido numerosos estudios comparativos a escala europea (Kikat, et al., 2007; Mönkediek \& Bras, 2018). Con tan pocos datos, determinar las causas de la baja fecundidad en España ha quedado a merced de la interpretación académica y de las teorías sobre la familia dominantes en cada momento. La publicación de la Encuesta de Fecundidad (EF) de 2018 pone fin a dos décadas sin una encuesta del Instituto Nacional de Estadística realizó. La anterior se realizó en 1999. Los investigadores sociales pueden ahora analizar las causas de la baja fecundidad en España sobre la base de una muestra representativa de 14.556 mujeres y 2.619 hombres.

En este artículo analizamos los principales resultados de esta encuesta. Empezamos examinando la evolución de la fecundidad de las generaciones de mujeres y hombres nacidos en España entre 1962 y 1989. Continuamos indagando sobre sus preferencias reproductivas en cuanto al tamaño ideal de familia y al deseo de tener hijos en los próximos tres años. Luego analizamos los motivos esgrimidos por los que mujeres y hombres no han alcanzado el número deseado de hijos y, a continuación, estimamos la fecundidad no realizada según sea el motivo principal que la ha impedido. Finalmente, utilizamos la información retrospectiva de la encuesta para analizar la asociación entre, por un lado, tener pareja o empleo estable y, por otro, la transición al primer hijo.

Más allá de la novedad que implica analizar unos datos tan recientes, la Encuesta de Fecundidad de 2018 posee tres atractivos adicionales. Primero, la encuesta recoge la experiencia de unas generaciones de mujeres y hombres muy homogéneas en cuanto a sus bajos niveles de fecundidad. Es, por tanto, una encuesta muy apropiada para estudiar la baja fecundidad. Segundo, la encuesta combina dos muestras independientes, una de mujeres y otra de hombres, que permite comparar resultados por sexo; algo poco habitual en los estudios de fecundidad, centrados mayoritariamente en la experiencia de las mujeres. Tercero, la encuesta pregunta directamente por las razones por las qué mujeres y hombres no han alcanzado, si es el caso, el número de hijos que deseaban. Las respuestas a esta pregunta informan de la variedad de motivos por los qué la gente no ha tenido los hijos que deseaba y de la importancia de estos motivos según la edad. Sobre esta información realizamos una propuesta para estimar la fecundidad no realizada, definida como la brecha entre la fecundidad observada y la fecundidad deseada cuando la primera es menor a la segunda (Casterline \& Han, 2016). Con este trabajo aspiramos a (i) comprender mejor la dinámica reproductiva de una de las poblaciones menos fecundas del mundo, (ii) discutir el encaje de España en las teorías de familia y (iii) aportar nuevas herramientas analíticas para el estudio de la baja fecundidad desde la perspectiva de la fecundidad no realizada. 


\section{Interpretaciones teóricas de la baja fecundidad y el encaje de España}

Cuando España cruzaba el umbral de los 1,3 hijos por mujer en 1991, la teoría de la segunda transición demográfica dominaba sobre los marcos interpretativos de la baja fecundidad en Europa (Van de Kaa, 1987; Lesthaeghe, 2010). Liderada por los países escandinavos, y dentro de éstos por la población más instruida, la caída de la fecundidad por debajo del nivel de reemplazo vino acompañada de un retraso de la edad a la unión, de un aumento de la cohabitación fuera del matrimonio y de una mayor inestabilidad de las uniones. Como principales agentes del cambio, la segunda transición demográfica enfatizó los factores ideacionales y culturales con el siguiente argumento: los procesos de individualización, asociados a los niveles crecientes de bienestar, modificarían las expectativas y las preferencias de los individuos alejándolos de los tiempos y formas de la familia tradicional. La transformación de los sistemas de valores y la tolerancia creciente hacia formas no tradicionales de familia reflejadas en las encuestas de valores avalarían esta interpretación (Surkyn \& Lesthaeghe, 2004; Inglehart, 2008; Kuyper et al., 2013). Si bien los cambios familiares esperados por la segunda transición demográfica se han generalizado a la mayoría de países occidentales (Kiernan, 2001; Sobotka, 2004), e incluso extendido a otras regiones del mundo (Raymo, et al., 2009; Esteve, et al., 2012; Yu \& Xie, 2015), la interpretación de sus causas ha sido cuestionada desde dos perspectivas.

La primera perspectiva crítica con la segunda transición demográfica argumenta que las causas materiales prevalecen sobre las ideacionales (Mills, et al. 2011; Kreyenfeld, 2010; Ruggles, 2015). La gente quiere, pero no puede tener hijos. La precariedad y la incertidumbre económica serían los principales obstáculos para formar pareja y tener hijos. A favor de esta interpretación tendríamos, primero, que el tamaño ideal de familia en países de baja fecundidad se ha mantenido en el tiempo en torno a los dos hijos (Sobotka, 2014; Hartnett, 2020) y, segundo, que las personas de nivel educativo alto y, normalmente, de mayor poder adquisitivo son las que, en algunas sociedades, tienen relaciones de pareja más estables y acaban incluso teniendo más hijos que las personas de menor nivel educativo (Jalovaara, et al., 2018). Una dinámica que reforzaría un patrón de desventaja social y de reproducción de desigualdades (PerelliHarris, et al., 2010).

La segunda perspectiva crítica con la segunda transición demográfica pone el acento en la relación entre las dinámicas familiares y la evolución de los roles de género en la sociedad (McDonald, 2000). El razonamiento es el siguiente: la irrupción de la mujer en el mercado laboral pondría en cuestión el modelo tradicional de pareja basado en la especialización de roles entre cónyuges dentro y fuera del hogar (Becker, 1973; Oppenheimer, 1988). Al no consolidarse un modelo alternativo nuevo, se abriría una etapa de inestabilidad poco favorable a la formación de parejas y a la crianza de hijos. Conforme se avanzaría en la igualdad de género y, más concretamente, conforme los hombres asumirían su parte de responsabilidad en las tareas reproductivas, se consolidaría un nuevo equilibrio de género y modelo familiar más favorable a la formación de parejas y a la fecundidad (Esping-Andersen \& Billari, 2015). Esta interpretación fue validada, y en parte inspirada, por la evolución de la fecundidad de las últimas dos décadas en países escandinavos. Tras diez años de caída continuada de la fecundidad, países como Suecia, Finlandia y Noruega experimentaron un repunte de la fecundidad que alimentó, entre otras, la teoría de la revolución de género (Goldscheider, et al., 2015). Sin embargo, estudios recientes muestran que la fecundidad ha vuelto a caer en estos países (Jalovaara, et al., 2019; Comolli, et al. 2020). Ante la débil o nula evidencia de una eventual recuperación de la fecundidad y el impacto de la gran recesión económica 
de 2008, ahora agravada por la crisis de la Covid-19, las explicaciones de corte material han vuelto a ganar fuerza (Seltzer, 2019; Matysiak, et al., 2020). Los jóvenes tienen dificultades para emanciparse de sus padres, acceder a la vivienda y encontrar empleos estables. La incertidumbre económica sacude sus vidas y compromete sus planes reproductivos (Vignoli et al., 2020).

Como representante de los países del sur de Europa, la posición de España en estos debates ha ido oscilando según variaban sus indicadores de fecundidad y de familia. A principios de los años $80 \mathrm{~s}$, al formularse los postulados de la segunda transición demográfica, España cumplía perfectamente con el rol de país tradicional. Su fecundidad era superior y más temprana que la de Suecia, el matrimonio era la vía hegemónica a la vida en pareja y la incidencia del divorcio era escasa (Alberdi, 1999). España era un ejemplo típico de país conservador, de raíz católica y con un fuerte apego a la familia. (Reher, 1998; Requena, 2005). A partir de la década de los noventa, sin embargo, se precipitaron cambios importantes en el ámbito de la familia. Aumentó la edad media a la primera unión y se retrasó la llegada del primer (Castro-Martín, 1992; Castro-Martín, 1993; Miret-Gamundi, 1997). La fecundidad cayó abruptamente (Castro-Martín \& Martín-García, 2013). Las parejas cohabitantes, las separaciones y los divorcios crecieron significativamente (Solsona \& Simó-Noguera, 2007; Domínguez-Folgueras \& Castro Martín, 2013; Bernardi \& Martínez-Pastor, 2011). Por nivel educativo, los más instruidos lideraron estas transiciones. Hay quien consideraba que España entraba de lleno en la segunda transición demográfica (Sobotka, 2008) y las encuestas de valores así lo reflejaban (Liebfroer \& Fokkema, 2008). Por ejemplo, el grado de tolerancia hacia formas familiares no tradicionales y el grado de aceptación de la homosexualidad o la eutanasia eran homologables a la de otros países occidentales, incluidos los nórdicos (Abou-Chadi \& Finnigan, 2019; Serrano \& Heredia, 2018).

Pero la caída de la fecundidad en España y su estancamiento en torno a los 1,3 hijos por mujer también encajaba con los postulados de la revolución de género (Goldscheider, et al., 2015). Por un lado, los avances en el ámbito educativo y laboral de la mujer en España son incuestionables. La proporción de mujeres jóvenes con estudios universitarios supera a la de los hombres de la misma franja de edad (Ortiz, Rodríguez-Menés, 2015; Esteve, et al., 2016) y la participación de las mujeres en el mercado laboral ha crecido (Guinea-Martín et al., 2018; Miret, 2018). Sin embargo, en el ámbito doméstico, la brecha de género en la dedicación a las tareas reproductivas es mayor en España que en otros países de la Europa occidental, especialmente los países escandinavos (Voicu, et al., 2009; Fahlén, 2016; Borràs et al., 2018). Los hombres españoles dedican dos horas menos a las tareas domésticas que las mujeres. La diferencia aumenta hasta las tres horas en las parejas con hijos (Moreno-Colom, 2017; Ajenjo \& García-Román, 2019). En países como Suecia o Noruega, la diferencia es de una hora (Moreno-Colom, 2017). La baja fecundidad en España es coherente por tanto con el avance de las mujeres en la esfera productiva y estancamiento de los hombres en la reproductiva.

Sin embargo, entre los académicos y expertos nacionales las razones materiales (ej. Desempleo, acceso a la vivienda, precariedad) predominan sobre todas las demás (Bernardi, et al., 2003; Castro-Martín \& Martín-García, 2016). Debido a la escasez de datos, es difícil establecer de forma objetiva las causas subyacentes de la baja fecundidad en España. La buena calidad de los datos del Movimiento Natural de Población utilizados para medir la fecundidad y la evolución de los nacimientos contrasta con la escasez de encuestas y datos retrospectivos y / o longitudinales. España no ha participado en el programa de la Generations and Gender y tampoco ha realizado encuestas de fecundidad regulares en el tiempo. En las 
últimas tres décadas, se han realizado tres encuestas, pero solo dos de ellas por el Instituto Nacional de Estadística, en 1999 y en 2018. En 2006 el Centro de Investigaciones Sociológicas realizó una encuesta de menor tamaño y que presentó algunos problemas técnicos en los ponderadores.

La escasez de datos contrasta con el elevado interés que, demográficamente hablando, tiene España como país de estudio. España es, junto a Italia, el país del mundo con una baja fecundidad más duradera en el tiempo. Cuenta, además, con la edad media al primer hijo más elevada de Europa y uno de los niveles de infecundidad más altos del mundo (Sobotka, 2017). Entre las mujeres nacidas a mediados de los años 70s, una de cada cuatro no será madre (Esteve, et al., 2016). Junto a la baja fecundidad, la disminución de la población en edad fértil está provocando la caída del número de nacimientos en España desde 2008. La crisis de la COVID-19 agravará esta situación. Las primeras estimaciones del INE apuntan a una caída del $20 \%$ de los nacimientos en los meses de diciembre de 2020 y enero y febrero 2021 respecto a los mismos meses un año antes. La caída de los nacimientos y la baja fecundidad inciden directamente en el nivel de envejecimiento de la población española. Naciones Unidas estima que un 36,8\% de la población tendrá más de 65 años de edad en 2050. Una cifra solo superada por Corea del Sur (38,1\%) y Japón (37,7\%).

La baja fecundidad en España se ha convertido en un elemento de debate político. Un debate agitado por grupos de presión que acusan indistintamente a los gobiernos de inacción y a los jóvenes de hedonistas (Macarrón, 2011). Lamentablemente, sin datos, el debate sobre las causas subyacentes de la baja fecundidad en España ha quedado a merced de la especulación académica y de creadores de opinión. La explotación de la Encuesta de Fecundidad de 2018 abre la puerta a investigar estas cuestiones y a mejorar la comprensión de un fenómeno con tres décadas de antigüedad y muy poca investigación al respecto. La estrategia analítica y resultados que presentamos a continuación pueden ser de utilidad para países que están en una situación similar (ej. Italia, Japón, Corea del Sur) y para todas aquellas que no alcanzan la fecundidad deseada. A partir del concepto de fecundidad no realizada proponemos una estrategia para medir la influencia de los distintos motivos por los que mujeres y hombres no han tenido los hijos que deseaban y su importancia en función de la edad. Con ello queremos mostrar la diversidad de factores que condicionan la baja fecundidad, identificarlos y medir su influencia y, en última instancia, guiar el desarrollo de la teoría.

\section{La Encuesta de Fecundidad de 2018: procesamiento y análisis de los datos}

En el año 2016 el Instituto Nacional de Estadística de España (INE) decidió realizar una nueva encuesta de fecundidad. El trabajo de campo se realizó entre los meses de enero y marzo de 2018 y los microdatos se publicaron en abril de 2019. La última encuesta de fecundidad realizada por el INE fue en 1999. Para elaborar el cuestionario se tuvieron en cuenta tres elementos: la comparabilidad con la encuesta de 1999, la homologación con otras encuestas europeas, especialmente la Generations and Gender Survey (GGS), y las recomendaciones de investigadores del Centro de Estudios Demográficos y del grupo de población del Consejo Superior de Investigaciones Científicas. El resultado fue una encuesta con más de 180 preguntas distribuidas en diez bloques temáticos ${ }^{1}$ y realizadas a 14.556 mujeres y a 2.619 hombres procedentes de dos muestras independientes. Se trata de una encuesta transversal que incluye preguntas

\footnotetext{
1 1) Identificación de la persona y datos biográficos, 2) Hogar, 3) Vivienda, 4) Familia de Origen, 5) Relaciones de pareja, 6) Hijos, 7) Recundidad, 8) Estudios, 9) Ocupación e Ingresos, 19) Valores y actitudes.
} 
retrospectivas sobre las trayectorias de pareja, fecundidad, vivienda y empleo de los entrevistados ${ }^{2}$. Este estudio se centra en las mujeres y hombres nacidos en España y residentes en este país en el momento de la encuesta. Se ha excluido a la población nacida en el extranjero por dos motivos. Primero porque no toda su fecundidad ha tenido lugar en España. Segundo porque el peso de la fecundidad de las extranjeras varía en función de las generaciones y esto dificulta la comparabilidad entre ellas. Al excluir las mujeres y hombres nacidos en el extranjero, el número final de casos ha quedado en 13.128 mujeres y 2.386 hombres. Los resultados han sido ponderados utilizando el factor de elevación que proporciona la encuesta.

En función de las variables analizadas, los resultados se presentan por cohortes de nacimiento o por edad en 2018. Hemos agrupado las cohortes en grupos de cinco generaciones empezando por la nacida entre 1965 y 1969 y acabando por la nacida entre 1985 y $1989^{3}$. Para cada cohorte se ha calculado la proporción de mujeres y hombres que ha tenido hijos y la fecundidad acumuladas por edad. Se ha considerado el total de hijos biológicos y adoptados tenidos hasta el momento de la encuesta. Los resultados se presentan por sexo y posteriormente por nivel educativo: bajo, medio y alto. El nivel bajo agrupa a todas las personas sin estudios y educación primaria (38\%). El nivel medio incluye a la población con estudios secundarios obligatorios y postobligatorios hasta bachiller $(26,3 \%)$. Y el nivel alto de la población con enseñanzas de formación profesional de grado superior y estudios universitarios $(35,7 \%)$.

Los deseos y expectativas de tener hijos se han medido en el momento de la encuesta. La encuesta pregunta por el tamaño ideal de familia y la edad ideal para tener un primer hijo. También pregunta por la intención de tener un hijo (más) en los próximos tres años ${ }^{4}$. Hemos utilizado esta información para captar el deseo de tener hijos en 2018. Los resultados se han desagregado esta información por paridad (0, 1, 2, o 3 y más hijos) y por edad simple (18 a 49). Al tratarse de generaciones muy homogéneas en cuanto a su bajo nivel de fecundidad (ver Figura 1), consideramos, con la debida prudencia, que los deseos expresados en 2018 pueden ser representativos de un periodo más amplio y del conjunto de cohortes analizadas en este trabajo, más aún sabiendo que el ideal de los dos hijos por mujer no ha variado en las últimas tres décadas (Sobotka y Beaujouan, 2014).

La encuesta pregunta por los motivos por los qué mujeres y hombres no han alcanzado el número de hijos deseados. Los entrevistados debían seleccionar de forma priorizada un máximo de tres opciones entre los 20 motivos pre-determinados por la encuesta y existía la posibilidad de añadir motivos adicionales. En el fichero de microdatos publicado por el INE aparecen 35 motivos. El 54,3\% de las mujeres indicaron un solo motivo, un $27,4 \%$ dos motivos y un $18,2 \%$ tres motivos. Hemos agrupado los motivos en seis categorías (ver Apéndice 1) según reflejaran: (1) el deseo de no tener (más) hijos; (2) el deseo de tenerlos más tarde; (3) no tener la pareja adecuada; (4) no reunir las condiciones materiales, económicas, y de conciliación para tener hijos; (5) problemas de salud para quedarse embarazada (6) y otros. Los resultados se analizan por edad, paridad y nivel educativo. A efectos de representación gráfica, las frecuencias han

\footnotetext{
${ }^{2}$ Existe una muestra de historias de vida basada en la información retrospectiva de la Encuesta de Fecundidad 2018 y armonizada según los criterios de la Generations and Gender Programme en [https://www.ggp-i.org/data/harmonized-histories/].

${ }^{3}$ Las cohortes entre 1962 y 1964 quedaron excluidas de la muestra por falta de muestra. Las mujeres nacidas entre 1965 y 1989 acumulan un $62.5 \%$ de la muestra.

${ }^{4}$ La pregunta original se formula de la siguiente manera: “¿Tiene la intención de tener uno o más hijos en los próximos 3 años? Y para las encuestadas embarazadas: "Además del hijo que espera, ¿tiene la intención de tener uno o más hijos en los próximos 3 años?
} 
sido suavizadas utilizando una función spline ${ }^{5}$ (Camarda, 2012). Solo hemos analizado el motivo principal. A partir de esta información hemos calculado la fecundidad no realizada. La fecundidad no realizada mide el impacto sobre la fecundidad de cada motivo, asumiendo que no hay otros motivos que lo impidan. Los resultados se presentan desagregados por sexo y nivel educativo. El Apéndice 2 describe el método de cálculo de la fecundidad no realizada.

Finalmente, utilizamos la información retrospectiva de la encuesta para examinar la transición al primer hijo de mujeres y hombres en función de su situación de pareja (si conviven o no en pareja) y laboral (si tienen o no un contrato laboral indefinido). Información disponible para 10,917 mujeres y 2,045 hombres ${ }^{6}$. Hemos reconstruido la historia de embarazos, de unión y laboral de trabajo para crear un fichero de personas-año. Para cada año identificamos si la persona vive en pareja, si tiene un contrato laboral indefinido y si tiene hijos. Seleccionamos a las mujeres y hombres que no han tenido hijos y calculamos la probabilidad de tener el primer hijo en los próximos tres años en función de su situación de pareja y laboral.

\section{Resultados}

\section{La fecundidad de las cohortes}

Los cuatro paneles de la Figura 1 muestran el porcentaje de madres / padres y la fecundidad acumuladas por edad de las generaciones de mujeres y hombres nacidos en España entre 1965 y 1989. Destacamos tres resultados. Primero, la proporción de personas que ha tenido al menos un hijo entre las cohortes de mujeres / hombres analizadas no supera el $75 \%$ del total y la fecundidad total acumulada en estas mismas cohortes no supera los 1,4 hijos por mujer / hombre. Segundo, el comportamiento de las cohortes es muy estable en el tiempo a excepción de la cohorte de mujeres nacida entre 1965-69, que empezó a tener hijos algo más temprano que el resto. Se trata, por tanto, de unas cohortes muy homogéneas en cuanto a su comportamiento reproductivo. Tercero, que la fecundidad de los hombres es a todas las edades y para todas las generaciones menor que la de las mujeres. La fecundidad de la cohorte nacida entre 1965 y 1969 es de 1,25 hijos para los hombres y de 1,40 para las mujeres.

Los datos de la Figura 2 muestran el porcentaje acumulado de mujeres y hombres que han sido madres y padres respectivamente por nivel educativo bajo, medio y alto. Las mujeres de mayor nivel educativo tienen los hijos más tarde que las mujeres con niveles medios o bajos de educación. La proporción de madres entre las mujeres de mayor nivel educativo es inferior a la del resto de grupo. El gradiente educativo se mantiene en todas las cohortes, aunque se acortan las distancias en algunas cohortes. Para la cohorte 1975-79, la proporción de mujeres de nivel educativo alto que son madres a los 35 años ha

\footnotetext{
5 Se han empleado dos técnicas de suavizado para generar las Figuras 3 y 4, a saber, la media móvil simple y el modelo P-spline. Primero, usamos el promedio ponderado (la media no ponderada de 9 puntos de datos) para resaltar la tendencia general de cada categoría (por ejemplo, Paridad 0, razones de pareja). En segundo lugar, utilizamos un modelo P-spline para suavizar cada categoría.

${ }^{6}$ Los datos han sido tratados de dos maneras distintas. Una primera de manera transversal, donde incluimos información de todas las mujeres y hombres que responden la encuesta, excluyendo los nacidos en el extranjero (13.128 mujeres y 2.386 hombres). La segunda reconstruye las historias de pareja, fecundidad y mercado laboral a través de las preguntas retrospectivas de la misma encuesta. Por ello perdemos un $16,8 \%$ de casos para las mujeres y 14,3\% entre los hombres. Esta pérdida de casos se debe a dos motivos principales. En primer lugar, hemos acotado la muestra a las personas nacidas entre 1965 y 1989. El número de casos entre las generaciones anteriores a 1965 eran muy pocos las nacidas después de 1989 son demasiado jóvenes para ser consideradas. En segundo lugar, la pérdida de casos se debe a la falta de información sobre fechas en que sucedieron los distintos eventos, no pudiendo así reconstruir la trayectoria. Hemos realizado test de sensibilidad para comprobar que la pérdida de casos no altere ni la magnitud ni el sentido de los resultados.
} 
alcanzado el nivel de las mujeres de nivel educativo medio a la misma edad. En los hombres, las diferencias por nivel educativo son más pequeñas que entre las mujeres. Los hombres de nivel educativo alto empiezan a tener hijos algo más tarde que el resto de grupos, pero las diferencias se igualan entre grupos a partir de los 40 años.

Figura 1. Proporción de madres y padres y fecundidad acumuladas por edad de las cohortes de mujeres y hombres nacidas en España entre 1965 y 1989
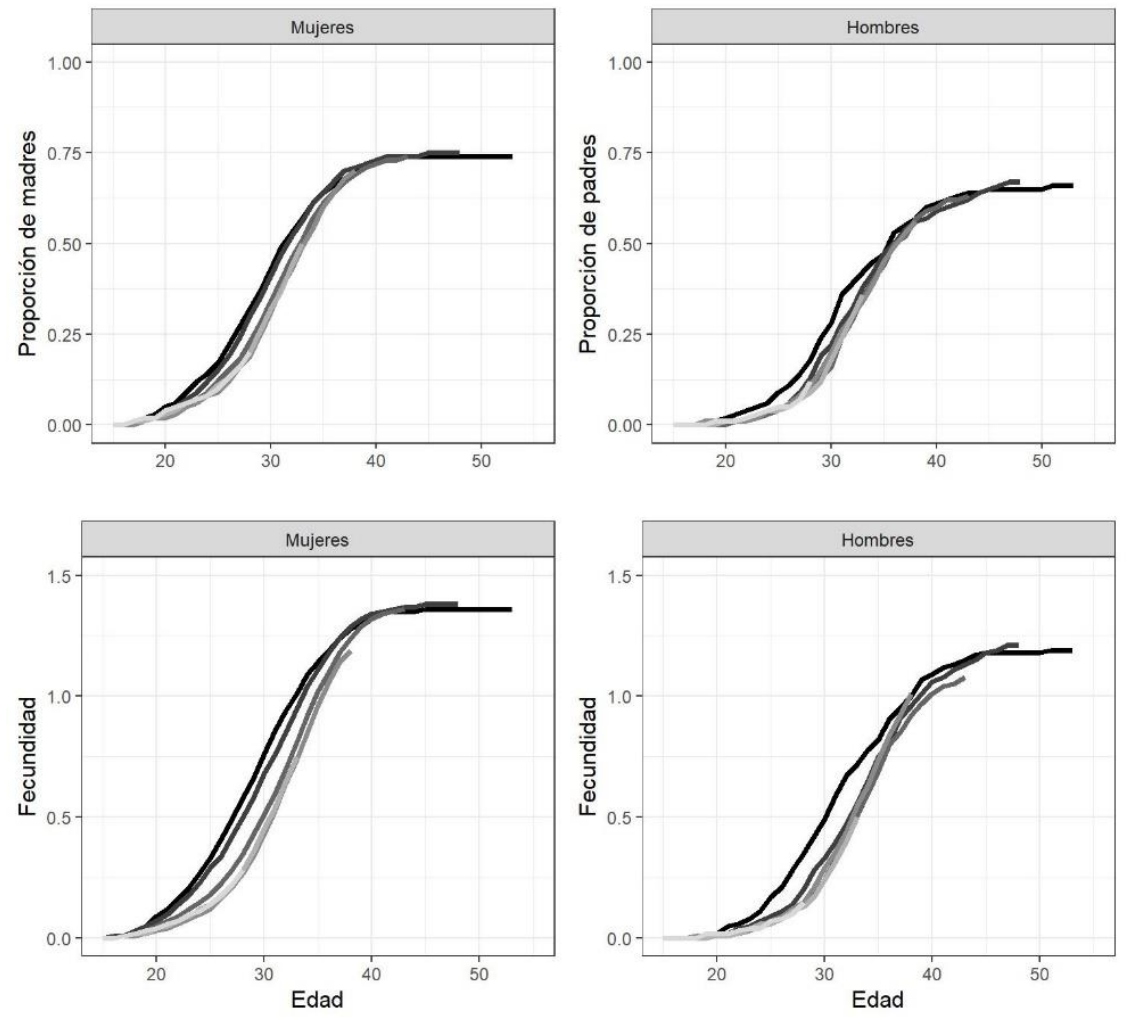

Cohorte de nacimiento $-{ }^{1962-1964-1965-1969-1970-1974-1975-1979-1980-1984}$

Fuente: Encuesta de Fecundidad de 2018, Instituto Nacional de Estadística.

\section{Intención de tener hijos en los próximos tres años}

La fecundidad en España está claramente por debajo de la fecundidad deseada por la población. La Figura 3 muestra el porcentaje de mujeres (panel izquierdo) y hombres (panel derecho) que expresaron en el momento de la encuesta el deseo de tener un hijo en los próximos tres años por edad y paridad (las paridades al momento de la entrevista están indicados con P1, P2 y P3). El color oscuro indica la intención de tener un hijo en los próximos tres años. El color claro indica lo contrario. En esta ocasión, no es posible utilizar información retrospectiva para reconstruir longitudinalmente las preferencias por tener hijos. Un 18,5 \% de las mujeres y un 20,7\% de los hombres expresaron la intención de tener un hijo en los próximos tres años. De haberse materializado estas intenciones, y asumiendo una distribución equitativa de los nacimientos en los tres años, la fecundidad de las mujeres nacidas en España en 2019 podría haber alcanzado los 2,3 hijos por mujer, casi el doble de la fecundidad observada en este mismo año para las mujeres nacidas en España $(1,17)$. 
Figura 2. Proporción acumulada de madres y padres por edad y nivel educativo de las cohortes de mujeres y hombres nacidas en España entre 1965 y 1989
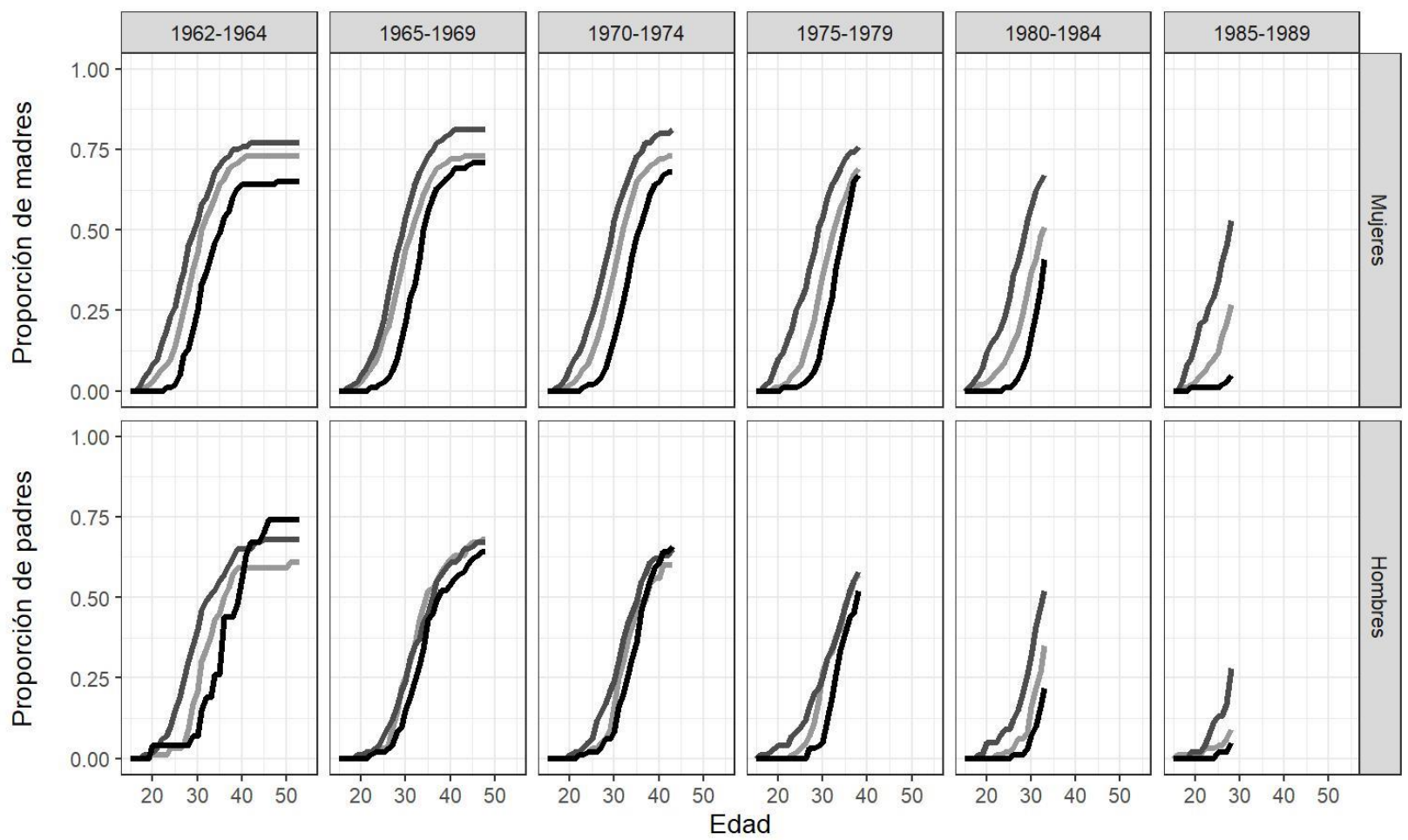

Nivel educativo - Bajo - Medio - Alto

Fuente: Encuesta de Fecundidad de 2018, Instituto Nacional de Estadística.

La intención de tener hijos varía en función del sexo, la edad y el número de hijos que tienen las personas. Hombres y mujeres presentan patrones por edad y paridad parecidos, aunque los niveles son ligeramente distintos. Antes de los 32 años, la proporción de mujeres que tiene intención de tener hijos es superior a la de los hombres. A partir de esta edad, la intención es mayor entre los hombres. A los 20 años de edad, menos de un $2 \%$ de mujeres y hombres tiene intención de tener hijos en los próximos tres años. El porcentaje aumenta rápidamente con la edad hasta aproximadamente los 40 años de edad. La mayoría de las personas que quieren tener hijos son personas sin hijos o con un hijo como máximo y de edades comprendidas entre los 28 y los 40 años. 
Figura 3. Intención de tener hijos en los próximos tres años por sexo, edad y paridad (P1, P2, P3) en el momento de la encuesta, 2018, de las mujeres nacidas en España

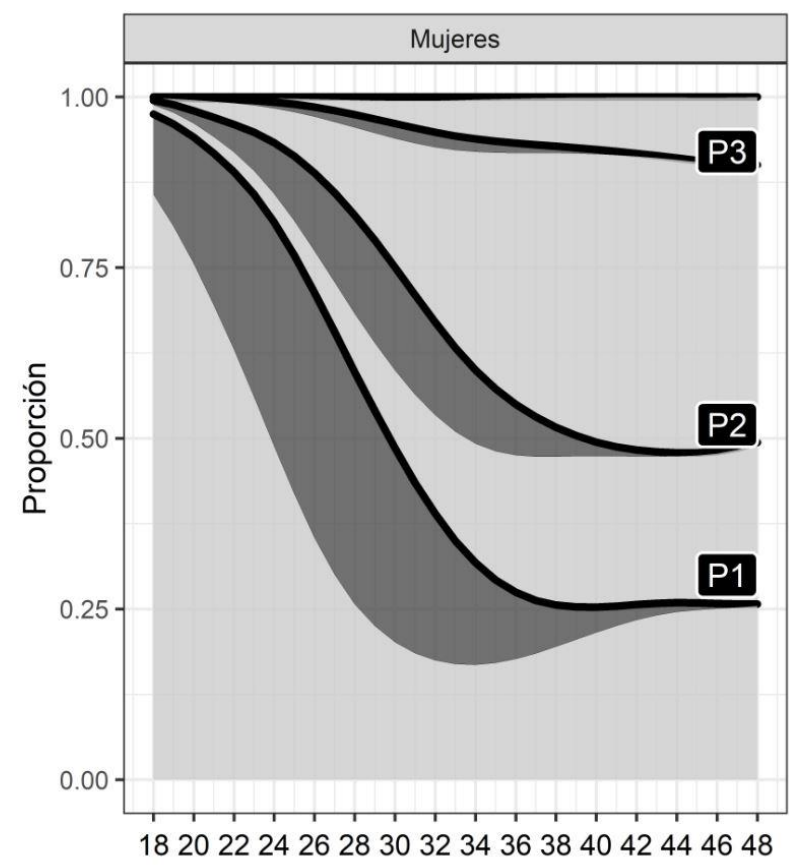

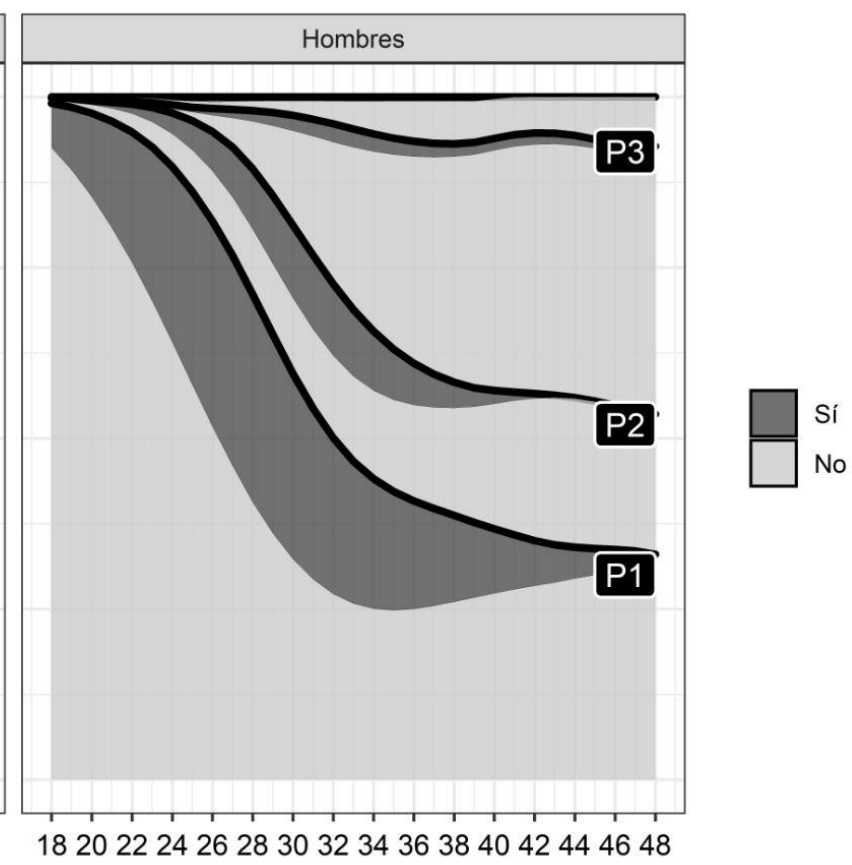

Edad

Fuente: Encuesta de Fecundidad, Instituto Nacional de Estadística 2018

\section{Motivos por los que no se han tenido los hijos que se deseaban y estimación de la fecundidad no realizada}

La Figura 4 muestra la distribución de los motivos por los cuales mujeres (panel izquierdo) y hombres (panel derecho) no han tenido los hijos que desearían tener por edad y paridad. También incluye a las personas que no quieren tener (más) hijos. La proporción de personas que no han tenido hijos y que no quiere tenerlos es inferior al $10 \%$ en todas las edades representadas. Antes de los 25 años, la mayoría de la población sin hijos se considera demasiado joven para tener hijos. A partir de esta edad, surgen otros motivos. Los motivos relacionados con la falta de pareja son especialmente importantes entre las personas sin hijos, y más en los hombres que en las mujeres. Los motivos materiales relacionados con la economía y la dificultad de conciliación son todavía más importantes que los de pareja y afectan principalmente a las personas entre 25 y 40 años que no han alcanzado el número deseado de hijos, personas normalmente sin hijos o con un solo hijo. A partir de los 40 años, los problemas de salud relacionados con la dificultad de quedarse embarazada tienen una incidencia mayor.

El cuadro 1 muestra la fecundidad no realizada según sea el motivo principal que la ha impedido. Se trata de una estimación hipotética según el método desarrollado en el Apéndice 2. Los resultados se muestran por sexo, paridad y nivel educativo de la población nacida en España que tenía entre 18 y 48 años de edad en 2018. Nos centramos en los motivos de pareja, los materiales y los de salud. No cuantificamos el efecto de 'no querer tener hijos' o 'ser demasiado jóvenes' al considerar que estos motivos reflejan las preferencias reproductivas de las personas y no las dificultades para alcanzar dichas preferencias. 
En esta cohorte sintética, la fecundidad de las mujeres $(1,39)$ es superior a las de los hombres $(1,21)$. Con los tres motivos juntos, la fecundidad no realizada es de 0,6 hijos para las mujeres y de 0,96 hijos para los hombres. Si sumamos estas cifras a la fecundidad observada, las mujeres nacidas en España alcanzarían los 2 hijos por mujer $(1,99)$ y los hombres superarían este umbral $(2,17)$. Los motivos materiales son los más importantes. La fecundidad no realizada por motivos materiales de 0,29 hijos por mujer y de 0,42 hijos por hombre. Entre los hombres, los motivos de pareja $(0,41)$ son igual de importantes que los materiales. Los motivos relacionados con la salud tienen un impacto sobre la fecundidad no realizada similar entre mujeres y hombres, en torno a los 0,12 hijos.

Figura 4. Motivos por los que no se han tenido los hijos que se deseaba por sexo, edad y paridad (P1, P2, P3) en el momento de la encuesta, 2018, de las mujeres nacidas en España

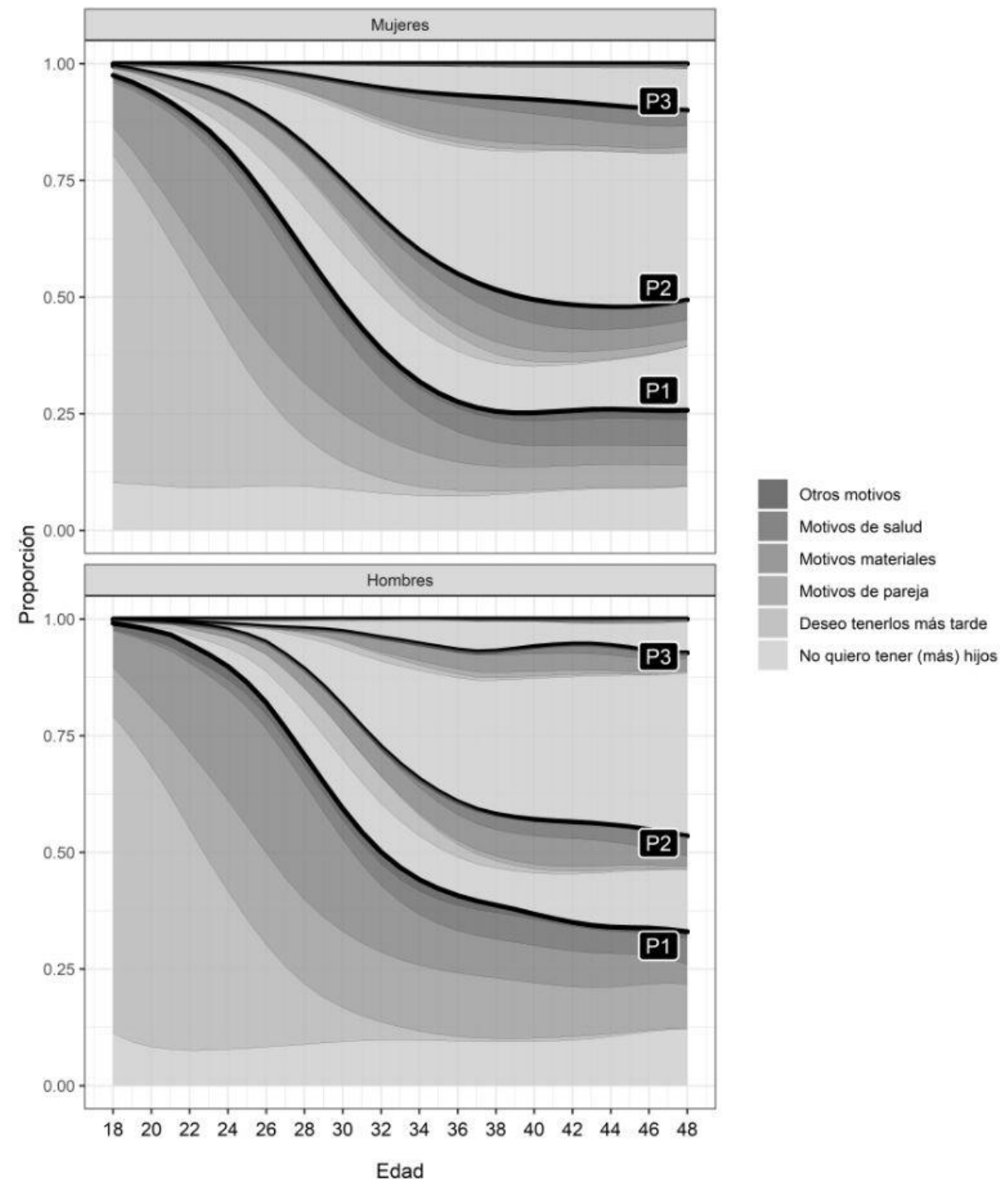

Fuente: Encuesta de Fecundidad de 2018, Instituto Nacional de Estadística. 
Por paridad, la fecundidad no realizada es más elevada en el segundo que en el primer hijo. Por ejemplo, los motivos materiales apenas impiden que mujeres y hombres tengan tarde o temprano un primer hijo, pero el impacto del retraso en el primer hijo reduce las probabilidades de tener un segundo hijo. Agrupando los tres motivos (pareja, materiales y salud), la fecundidad no realizada de las mujeres en relación con el segundo hijo es prácticamente el doble $(0,25)$ que en relación con el primer hijo $(0,13)$.

La fecundidad no realizada de las mujeres aumenta con el nivel de instrucción debido a la influencia que tienen los motivos materiales y de la pareja entres las mujeres de mayor nivel educativo. La fecundidad no realizada es de 0,70 hijos por mujer de nivel alto de instrucción y de 0,5 por mujer con nivel bajo de instrucción. Los hombres muestran un patrón distinto. La fecundidad no realizada es más alta en los extremos de la jerarquía educativa, pero por motivos distintos. Los motivos de pareja prevalecen sobre los materiales entre los hombres de nivel educativo bajo y, por el contrario, los materiales prevalecen sobre los de pareja entre los hombres de nivel educativo alto.

Cuadro 1. Fecundidad no realizada según sea el motivo principal que la ha impedido por sexo, paridad y nivel educativo

\begin{tabular}{|c|c|c|c|c|c|c|c|c|c|c|}
\hline & \multicolumn{5}{|c|}{ Mujeres } & \multicolumn{5}{|c|}{ Hombres } \\
\hline & \multirow{2}{*}{$\begin{array}{c}\text { Fecundidad } \\
\text { Observada }\end{array}$} & \multicolumn{4}{|c|}{ fecundidad no realizada debido a razones de... } & \multirow{2}{*}{$\begin{array}{l}\text { Fecundidad } \\
\text { Observada }\end{array}$} & \multicolumn{4}{|c|}{ fecundidad no realizada debido a razones de... } \\
\hline & & Pareja & Materiales & Salud & Todas & & Pareja & Materiales & Salud & Todas \\
\hline \multicolumn{11}{|l|}{ Todos } \\
\hline Paridad 0 & 0,76 & 0,04 & 0,03 & 0,06 & 0,13 & 0,68 & 0,10 & 0,05 & 0,05 & 0,21 \\
\hline Paridad 1 & 0,53 & 0,08 & 0,13 & 0,05 & 0,25 & 0,47 & 0,14 & 0,14 & 0,05 & 0,32 \\
\hline Paridad 2+ & 0,09 & 0,06 & 0,13 & 0,02 & 0,22 & 0,07 & 0,17 & 0,23 & 0,02 & 0,43 \\
\hline Total & 1,39 & 0,19 & 0,29 & 0,12 & 0,60 & 1,21 & 0,41 & 0,42 & 0,12 & 0,96 \\
\hline \multicolumn{11}{|c|}{ Nivel educativo bajo } \\
\hline Paridad 0 & 0,80 & 0,03 & 0,03 & 0,06 & 0,12 & 0,67 & 0,10 & 0,05 & 0,06 & 0,21 \\
\hline Paridad 1 & 0,55 & 0,08 & 0,09 & 0,04 & 0,21 & 0,44 & 0,18 & 0,12 & 0,05 & 0,34 \\
\hline Paridad 2+ & 0,10 & 0,07 & 0,08 & 0,02 & 0,17 & 0,08 & 0,20 & 0,17 & 0,04 & 0,40 \\
\hline Total & 1,45 & 0,18 & 0,19 & 0,12 & 0,50 & 1,19 & 0,47 & 0,34 & 0,15 & 0,94 \\
\hline \multicolumn{11}{|c|}{ Nivel educativo medio } \\
\hline Paridad 0 & 0,74 & 0,05 & 0,04 & 0,06 & 0,14 & 0,66 & 0,11 & 0,07 & 0,04 & 0,22 \\
\hline Paridad 1 & 0,49 & 0,08 & 0,14 & 0,04 & 0,26 & 0,45 & 0,13 & 0,14 & 0,04 & 0,31 \\
\hline Paridad 2+ & 0,06 & 0,06 & 0,15 & 0,01 & 0,24 & 0,07 & 0,08 & 0,15 & 0,02 & 0,25 \\
\hline Total & 1,29 & 0,19 & 0,33 & 0,11 & 0,63 & 1,17 & 0,32 & 0,36 & 0,10 & 0,78 \\
\hline \multicolumn{11}{|c|}{ Nivel educativo alto } \\
\hline Paridad 0 & 0,72 & 0,06 & 0,04 & 0,05 & 0,15 & 0,68 & 0,10 & 0,06 & 0,04 & 0,20 \\
\hline Paridad 1 & 0,52 & 0,10 & 0,15 & 0,05 & 0,29 & 0,54 & 0,12 & 0,13 & 0,06 & 0,30 \\
\hline Paridad 2+ & 0,10 & 0,07 & 0,16 & 0,02 & 0,26 & 0,06 & 0,14 & 0,28 & 0,02 & 0,43 \\
\hline Total & 1,34 & 0,23 & 0,35 & 0,13 & 0,70 & 1,28 & 0,35 & 0,47 & 0,12 & 0,93 \\
\hline
\end{tabular}

Fuente: Encuesta de Fecundidad, Instituto Nacional de Estadística 2018

\section{Determinantes próximos de la transición al primer hijo}

Dada la importancia que tienen los motivos de pareja y materiales sobre la fecundidad no realizada, utilizamos la información retrospectiva de la encuesta para analizar si la situación de pareja y laboral de las mujeres y hombres sin hijos está relacionada con la probabilidad de tener un hijo en los próximos tres años (ver Figura 5). Para ello, retomamos la perspectiva de cohorte y volvemos a los datos retrospectivos. En relación con la pareja, distinguimos entre los que vivían en pareja y los que no. Respecto a la situación 
laboral, clasificamos a las personas según tenían un contrato de trabajo permanente, un contrato temporal, o no tenían trabajo.

Figura 5. Probabilidad de tener un primer hijo en los próximos tres años en función de la situación de pareja y laboral a cada edad de las mujeres y hombres nacidos en España entre 1965 y 1989
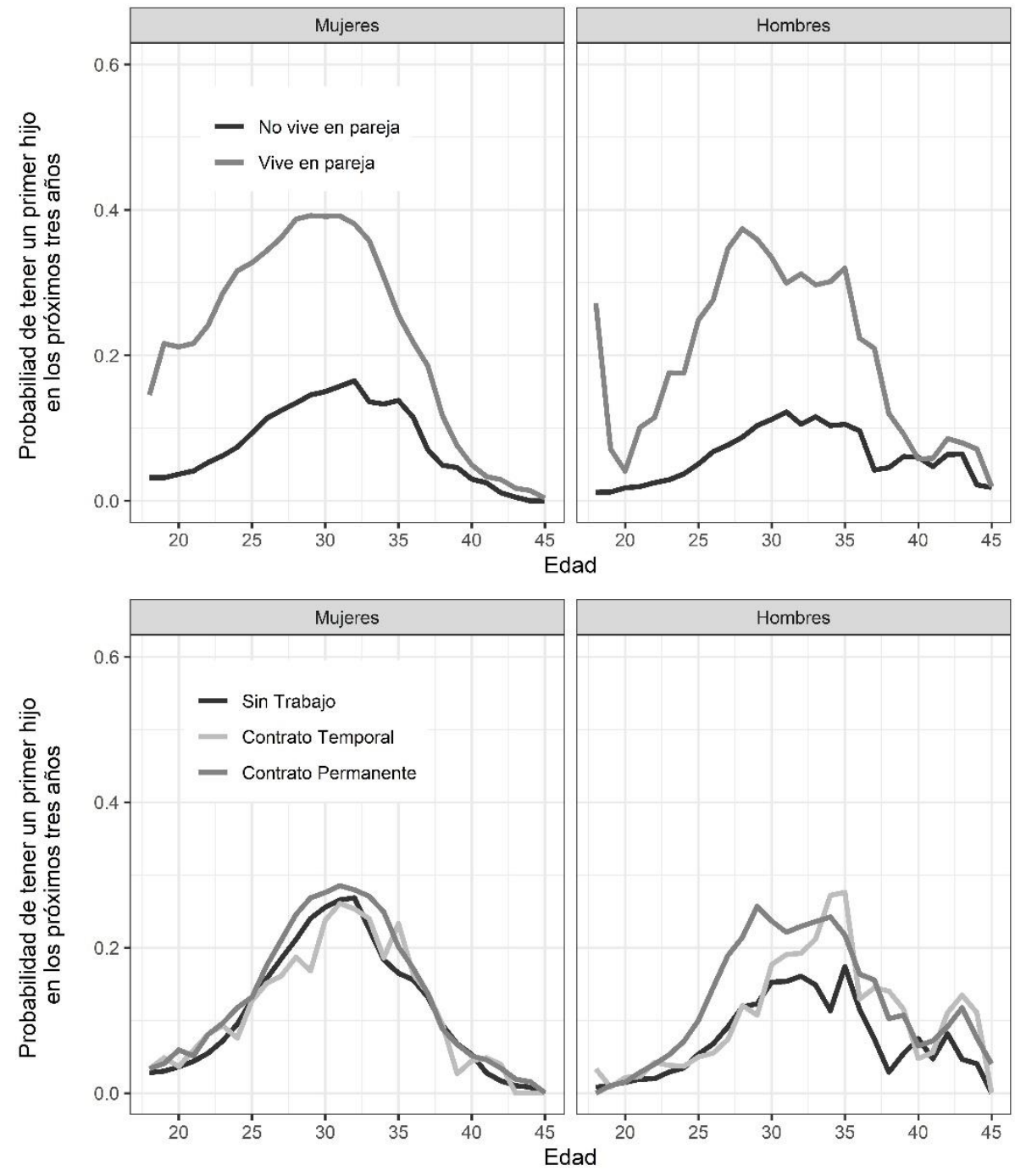

Fuente: Encuesta de Fecundidad, Instituto Nacional de Estadística 2018. Nota: El número de casos entre hombres es mucho menor al de las mujeres, y al dividir la muestra por cohortes de nacimiento el número de casos se reduce aún más. La estructura de los datos es lo que explica el cambio de la probabilidad entre los 18 y los 20 años para los hombres cohabitantes.

La probabilidad de tener un primer hijo varía en función de la edad. El riesgo es relativamente bajo antes de los 20 años, aumenta hasta alcanzar su máximo nivel entre los 30 y 35 años de edad y decrece rápidamente a partir de esa edad. Tener y convivir con la pareja aumenta la probabilidad de tener un primer hijo en mujeres y en hombres de edades comprendidas entre los 20 y los 40 años. Dentro de esta franja, la importancia de vivir en pareja sobre la probabilidad de tener un primer hijo es mayor entre los 25 y 35 años de edad. Por el contrario, la situación laboral de la mujer no es una variable para explicar la 
transición al primer hijo, incluso teniendo en cuenta si vive o no vive en pareja (resultados no mostrados). En cambio, el riesgo de tener un hijo en los próximos tres años es mayor entre los hombres que tienen un contrato permanente que entre los que no tienen trabajo o tienen un contrato temporal. No obstante, tanto para hombres como para mujeres, la estabilidad en el empleo sí que está relacionada con el inicio de la vida en pareja (resultados no mostrados).

\section{Resumen y discusión final}

En 2021, España cumplió 30 años desde que, por primera vez en su historia, la fecundidad se situara por debajo de los 1,3 hijos por mujer. Desde entonces, la fecundidad de las mujeres nacidas en España ha estado siempre por debajo de este umbral. En consecuencia, España es hoy uno de los países más envejecidos del mundo. Sin embargo, la baja fecundidad no impidió que los nacimientos aumentaran entre 1998 y 2008 y tampoco que la población creciera gracias a una estructura demográfica favorable a la natalidad y a la llegada de más de siete millones de inmigrantes internacionales. Estas tendencias contribuyeron a que la baja fecundidad no fuera percibida como un reto por la sociedad. Sin embargo, la caída del número de nacimientos desde el año 2009, la ralentización del crecimiento de la población y el hecho de que la cifra de muertes superara a la de nacimientos en 2016 por primera vez en 80 años generó las condiciones necesarias para que el Instituto Nacional de Estadística realizara una nueva encuesta de fecundidad. El trabajo de campo se realizó en 2018 y los resultados se publicaron en 2019. De la explotación de esta encuesta, destacamos cuatro grandes conclusiones.

Primero, se constata el carácter estructural de la baja fecundidad en España. Las cohortes analizadas son muy homogéneas en cuanto a su baja fecundidad y no hay atisbo de recuperación entre las más jóvenes. Los hombres tienen una fecundidad menor a la de las mujeres. Segundo, la fecundidad observada está claramente por debajo de la fecundidad deseada. Si una de cada tres mujeres que en 2018 manifestó la intención de tener hijos en los próximos tres años se hubiera reproducido en 2019, la fecundidad en España hubiera alcanzado los 2,3 hijos por mujer; casi el doble de los 1,17 hijos por mujer registrados por el INE en ese mismo año. Tercero, los motivos materiales relacionados con las dificultades económicas y de conciliación son los principales responsables de que mujeres y hombres no alcancen el número de hijos deseados y éstos prevalecen sobre los motivos de pareja y de salud. La fecundidad no realizada por todos estos motivos es de 0,6 hijos por mujer y de 0,96 hijos por hombre. La mayor parte de la fecundidad no realizada se observa en el segundo hijo. Cuarto, el análisis retrospectivo muestra la importancia de convivir en pareja entre los 25 y 35 años como determinante próximo de la fecundidad. El empleo estable de las mujeres no es un determinante próximo de la fecundidad, pero sí que lo es para entrar a vivir en pareja.

Desde el punto de vista metodológico, en este artículo hemos propuesto e implementado una estrategia para medir la fecundidad no realizada analizando los motivos por los que mujeres y hombres no alcanzan el número de hijos que desean. La naturaleza transversal de los datos y la dificultad de medir la simultaneidad y complementariedad entre motivos condiciona la interpretación de los resultados. Sin embargo, estos resultados son útiles para cuantificar la importancia de estos motivos en términos de fecundidad no realizada y, eventualmente, para guiar la acción política. Nuestra estrategia de análisis evoca el marco de los determinantes próximos de la fecundidad (Bongaarts, 1978). Los demógrafos tenemos herramientas y datos para, sin perder la perspectiva poblacional, analizar las razones por las que 
las personas no tienen hijos en cada momento de sus vidas reproductivas. El método que proponemos tiene la ventaja de captar la influencia de cada motivo a cada edad. Los resultados ponen en evidencia que las explicaciones basadas en un solo factor son insuficientes para entender las diferencias de fecundidad entre países. Las encuestas de fecundidad siguen siendo el instrumento más potente que tenemos los demógrafos para aproximarnos al reto de la baja fecundidad. Por este motivo, es necesario insistir en su realización ya sea a través de los Institutos Nacionales de Estadística o apoyando programas como el Generations and Gender Survey. Gracias a estas encuestas, el estudio comparativo de las causas de la baja fecundidad podría generalizarse al conjunto de Europa en un contexto de caída generalizada de la fecundidad ahora agravada por la crisis de la Covid-19. Aunque el deseo de tener hijos se mantenga, la población en edad fértil, especialmente la más joven no reúne las condiciones necesarias para ser padres. La edad a la que alcanzan estas condiciones es clave para poder alcanzar, dentro de los límites impuestos por la biología, el tamaño ideal de familia. Nuestros resultados apuntan que los factores ideacionales, típicamente asociados a la segunda transición demográfico, estarían explicando el porqué las personas no se plantean tener hijos antes de los treinta años, pero, a partir de esta edad, los factores materiales y de pareja juegan un papel determinante. Probablemente, los factores materiales también estén incidiendo en la formación de la pareja, especialmente entre los hombres y, entre ellos, los que tienen un nivel de instrucción más bajo. Los hombres de mayor nivel educativo no tendrían tantos problemas para emparejarse, pero en cambio aluden a motivos de orden material para tener hijos.

Más allá de las aportaciones de este trabajo al estudio de la fecundidad, y volviendo al caso España, las conclusiones del mismo tienen implicaciones relevantes para la acción política. En cuanto a sus preferencias reproductivas, España no es un país distinto al de resto de países de Europa (Sobotka \& Beaujouan, 2014). Sin embargo, la sociedad española no aprovecha todo su potencial reproductivo. Los jóvenes se emancipan tardíamente de sus padres, la vida en pareja se inicia también tarde y esto acorta el tiempo que lo jóvenes tienen para alcanzar sus deseos reproductivos. La precariedad laboral, el desempleo y la incertidumbre económica limitan la capacidad de estos jóvenes españoles para emanciparse. Sin políticas públicas de promoción a la emancipación y de inserción laboral de los jóvenes o, en su defecto, sin un mercado laboral dinámico, sin desempleo y con buenos salarios, los jóvenes españoles no alcanzan a edades tempranas la seguridad necesaria para tener los hijos que desean. La transición a la vida en pareja también se ve afectada por las condiciones precarias de los jóvenes. La crisis de la COVID-19 no hará otra cosa que agravar las condiciones en las que se reproducen los jóvenes. Los primeros datos disponibles muestran una caída de los nacimientos superior al $20 \%$ respecto al año anterior para los meses de diciembre 2020, y enero y febrero 2021. Y estos probablemente sean solo los efectos más inmediatos. Retrasar la fecundidad sobre un calendario ya de por sí tardío tendrá consecuencias irreversibles en la proporción de mujeres y hombres que no alcanzarán a tener ningún hijo y aumentará la brecha entre la fecundidad deseada y la fecundidad observada.

\section{Referencias}

Abou-Chadi, T., Finnigan, R. (2019). Rights for same-sex couples and public attitudes toward gays and lesbians in Europe. Comparative Political Studies, 52(6), 868-895.

Ajenjo, M., García-Román, J. (2019). La persistente desigualdad de género en el uso del tiempo en España. Perspectives Demogràfiques, 014, 1-4. 
Alberdi, I. (1999). La nueva familia española (Vol. 10). Madrid: Taurus.

Baizán, P., Aassve, A., \& Billari, F. C. (2003). Cohabitation, marriage, and first birth: The interrelationship of family formation events in Spain. European Journal of Population/Revue européenne de Démographie, 19(2), 147-169.

Becker, G. S. (1973). A theory of marriage: Part I. Journal of Political Economy, 81(4), 813-846.

Bernardi, F., \& Martínez-Pastor, J. I. (2011). Divorce risk factors and their variations over time in Spain. Demographic Research, 24, 771-800.

Bernardi, F., Requena, M., \& de Revenga, D. (2003). La caída de la fecundidad y el déficit de natalidad en España. RES. Revista Española de Sociología, 3, 29-49.

Bongaarts, J. (1978). A Framework for Analyzing the Proximate Determinants of Fertility. Population and Development Review, 4 (1), 105- 32.

Borràs, V., Ajenjo, M., \& Moreno-Colom, S. (2018). More time parenting in Spain: A possible change towards gender equality? Journal of Family Studies, https://doi.org/10.1080/13229400.2018.1440618

Camarda, C.G. (2012). Mortality Smooth: An R package for smoothing Poisson counts with P-Spline. Journal of Statistical Software, 50(1), 1-24.

Casterline, J. B., \& Han, S. (2017). Unrealized fertility: Fertility desires at the end of the reproductive career. Demographic research, 36, 427-454.

Castro-Martin, T. (1992). Delayed childbearing in contemporary Spain: trends and differentials. European Journal of Population/Revue Européenne de Démographie, 8(3), 217-246.

Castro-Martin, T. (1993). Changing nuptiality patterns in contemporary Spain. Genus, 49(1-2), 79-95.

Castro-Martín, T. y T. Martín-García (2013), Fecundidad bajo mínimos en España: pocos

hijos, a edades tardías y por debajo de las aspiraciones reproductivas, en G. Esping-Andersen

(Coord.), El déficit de la natalidad en Europa. La singularidad del caso español, Barcelona: Obra Social

La Caixa, 48-88.

Castro-Martín, T.; Martín-García, T. (2016). La fecundidad en España: entre las más bajas del mundo y sin muchas perspectivas de recuperación, Panorama social 23, 11-26.

Comolli, C.L., Neyer, G., Andersson, G. et al. (2020). Beyond the Economic Gaze: Childbearing During and After Recessions in the Nordic Countries. European Journal of Population, https://doi.org/10.1007/s10680-020-09570-0

Esping-Andersen, G., \& Billari, F. C. (2015). Re-theorizing family demographics. Population and Development Review, 41(1), 1-31.

Esteve, A., Schwartz, C. R., Van Bavel, J., Permanyer, I., Klesment, M., \& Garcia, J. (2016). The end of hypergamy: Global trends and implications. Population and Development Review, 42(4), 615.

Esteve, A., Devolder, D., Domingo, A., (2016) Childlessness in Spain: Tick Tock, Tick Tock, Tick Tock!, Perspectives Demogràfiques, 1, 1-4. 
Fahlém, S. (2016). Equality at home - A question of career? Housework, norms, and policies in a European comparative perspective. Demographic Research, 35(48), 1441-1440.

Goldscheider, F., Bernhardt, E., Lappegard, T. (2015). The gender revolution: A framework for understanding changing family and demographic behaviour. Population and Development Review, 41(2), 207-239.

Guinea-Martin, D., Mora, R., \& Ruiz-Castillo, J. (2018). The Evolution of Gender Segregation over the Life Course. American Sociological Review, 83(5), 983-1019.

Hartnett, C. S., \& Gemmill, A. (2020). Recent trends in US childbearing intentions. Demography, 57(6), 2035-2045.

Inglehart, R. (2008). Changing values among western publics from 1970 to 2006. West European Politics, 31(1-2), 130-146.

Jalovaara , M., Neyer, G., Andersson, G., Dahlberg, J., Dommermuth, L., Fallesen, P., Lappegard, T. (2019). Education, Gender, and Cohort Fertility in the Nordic Countries. European Journal of Population, 35, 563-586.

Kohler, H. P., Billari, F. C., \& Ortega, J. A. (2002). The emergence of lowest-low fertility in Europe during the 1990s. Population and Development Review, 28(4), 641-680.

Kiernan, K. (2001). The rise of cohabitation and childbearing outside marriage in Western Europe. International journal of law, policy and the family, 15(1), 1-21.

Kreyenfeld, M. (2010). Uncertainties in female employment careers and the postponement of parenthood in Germany. European sociological review, 26 (3), 351-366.

Kuyper, L., Iedema, J., \& Keuzenkamp, S. (2013). Towards tolerance. Exploring changes and explaining differences in attitudes towards homosexuality in Europe. The Hague, the Netherlands: Netherlands Institute for Social Research (SCP).

Lesthaeghe, R. (2010). The unfolding story of the second demographic transition. Population and Development Review, 36(2), 211-251.

Liefbroer, A. C., \& Fokkema, T. (2008). Recent trends in demographic attitudes and behaviour: Is the Second Demographic Transition moving to Southern and Eastern Europe? In J. Surkyn, J. v. Bavel, \& P. Deboosere (Eds.), Demographic challenges for the 21st century. A state of art in demography (pp. 115-141). Brussels: Brussels University Press.

Macarrón, A. (2011). El suicidio demográfico de España. Madrid: Homo Legens, SL.

Matysiak, A., Sobotka, T., \& Vignoli, D. (2020). The Great Recession and fertility in Europe: A subnational analysis. European Journal of Population, 1-36. https://doi.org/10.1007/s10680-020-09556-y

McDonald, P. (2000). Gender equity in theories of fertility transition. Population and Development Review, 26 (3), 427-439.

Mills, M., Rindfuss, R. R., McDonald, P., \& Te Velde, E. (2011). Why do people postpone parenthood? Reasons and social policy incentives. Human reproduction update, 17 (6), 848-860.

Miret-Gamundi, P. (1997). Nuptiality patterns in Spain in the eighties. Genus, 53 (3-4), 183-198. 
Miret, P. (2018), Composición demográfica de la fuerza de trabajo y el empleo, Gaceta Sindical, Reflexión y debate (Distribución de la renta, desigualdades y brechas sociales), 31: 271-283.

Mönkediek, B., \& Bras, H. (2018). Family systems and fertility intentions: Exploring the pathways of influence. European Journal of Population, 34 (1), 33-57.

Moreno-Colom, S. (2017). The gendered division of housework time: Analysis of time use by type and daily frequency of households tasks. Time and Society, 26 (1), 3-27.

Morgan, S. P., \& Rackin, H. (2010). The correspondence between fertility intentions and behavior in the United States. Population and Development Review, 36 (1), 91-118.

Oppenheimer, V. K. (1988). A theory of marriage timing. American Journal of Sociology, 94(3), 563591.

Ortiz, L., Rodríguez-Menés, J. (2015). The positional value of education and its effect on general and technical fields of education: educational expansion and occupational returns to education in Spain. European Sociological Review, 32 (2), 216-237.

Perelli-Harris, B., Sigle-Rushton, W., Kreyenfeld, M., Lappegård, T., Keizer, R., \& Berghammer, C. (2010). The educational gradient of childbearing within cohabitation in Europe. Population and Development Review, 36 (4), 775-801.

Raymo, J. M., Iwasawa, M., \& Bumpass, L. (2009). Cohabitation and family formation in Japan. Demography, 46(4), 785-803.

Requena, M. (2005). The secularization of Spanish society: Change in religious practice. South European Society and Politics, 10 (3), 369-390.

Ruggles, S. (2015). Patriarchy, power, and pay: The transformation of American families, 1800-2015. Demography, 52 (6), 1797-1823.

Seltzer, N. (2019). Beyond the Great Recession: Labor market polarization and ongoing fertility decline in the United States. Demography, 56 (4), 1463-1493.

Serrano del Rosar, R., Heredia Cerro, A. (2018). Spanish attitudes towards euthanasia and physicianassisted suicide. Revista Española de Investigaciones Sociológicas, 161, 103-120.

Sobotka, T. (2004). Postponement of childbearing and low fertility in Europe (pp. 298-pp). Amsterdam: Dutch University Press.

Sobotka, T. (2008). Overview chapter 6: The diverse faces of the second demographic transition in Europe. Demographic research, 19, 171-224.

Sobotka, T., \& Beaujouan, É. (2014). Two Is best? The persistence of a two-child family ideal in Europe. Population and Development Review, 40(3), 391-419.

Sobotka, T. (2017). Childlessness in Europe: Reconstructing long-term trends among women born in 1900-1972. In M. Kreyenfeld \& D. Konietzka (Eds.), Childlessness in Europe: Contexts, causes, and consequences. Berlin: Springer, 17-53.

Solsona, M., Simó-Noguera, C. (2007). Evolución histórica del divorcio en España desde la aprobación de la ley de 1981 hasta la reforma de 2004. In A. Cabré \& P Miret (Eds.), La constitución familiar en España. Bilbao: Fundación BBVA, 245-296. 
Surkyn, J., \& Lesthaeghe, R. (2004). Value orientations and the second demographic transition (SDT) in Northern, Western and Southern Europe: An update. Demographic research, 3, 45-86.

Van de Kaa, D. J. (1987). Europe's second demographic transition. Population bulletin, 42(1), 1-59.

Vignoli D., Bazzani G., Guetto R., Minello A., Pirani E. (2020) Uncertainty and Narratives of the Future: A Theoretical Framework for Contemporary Fertility. In: Schoen R. (eds) Analyzing Contemporary Fertility. The Springer Series on Demographic Methods and Population Analysis, vol 51. Springer, Cham. https://doi.org/10.1007/978-3-030-48519-1_3

Vikat, A., Spéder, Z., Beets, G., Billari, F. C., Bühler, C., Désesquelles, A., ... \& Sola, A. (2007). Generations and Gender Survey (GGS) Towards a better understanding of relationships and processes in the life course. Demographic research, 17, 389-440.

Voicu, M., Voicu, B., Strapcova, K. (2009). Housework and gender inequality in European countries. European Sociological Review, 25(3), 365-377.

Yu, J., \& Xie, Y. (2015). Cohabitation in China: Trends and determinants. Population and Development Review, 41(4), 607-628.

Agradecimientos: A Daniel Devolder por sus consejos en la elaboración de este artículo y cálculo de indicadores. A Anna Turu por la preparación de los gráficos. Este trabajo ha recibido la financiación del Ministerio de Ciencia, Innovación y Universidades (RTI2018-096730-B-I00). 


\section{Apéndice 1. Clasificación de los motivos por los cuales no se ha alcanzado el número de hijos deseado hasta la fecha}

Agrupación de razones por las que las mujeres entrevistadas indican no tener los hijos deseados y peso (en porcentaje) de cada motivo según paridad $(0,1,2,3)$ - Generaciones de mujeres nacidas en España entre 1965 y 1989.

\begin{tabular}{|c|c|c|c|c|}
\hline \multirow[b]{2}{*}{ Respuestas } & \multicolumn{4}{|c|}{ Paridad } \\
\hline & 0 & 1 & 2 & $3+$ \\
\hline \multicolumn{5}{|l|}{ No quiero tener (más) hijos } \\
\hline $\begin{array}{l}\text { Los embarazos, partos y cuidado de los hijos son duros para la } \\
\text { mujer }\end{array}$ & 0,20 & 1,04 & 1,00 & 1,56 \\
\hline No quiero ser madre & 5,54 & & & \\
\hline $\begin{array}{l}\text { Porque supone perder libertad y no tener tiempo para realizar } \\
\text { otras actividades }\end{array}$ & 0,7 & 0,42 & 0,42 & 0,35 \\
\hline \multicolumn{5}{|l|}{ Motivos juventud o deseo de tenerlos más tarde } \\
\hline Soy demasiado jóven para tener hijos & 71,49 & & & \\
\hline Todavía quiero tener más hijos pero no ahora & & 20,48 & 4,80 & 3,01 \\
\hline Deseo seguir estudiando & 2,86 & 0,08 & 0,10 & 0,09 \\
\hline \multicolumn{5}{|l|}{ Motivos de pareja } \\
\hline Mi pareja no ha querido tener hijos/No quiere todavía & 0,34 & 3,25 & 6,20 & 7,22 \\
\hline No tengo pareja o ésta no es adecuada & 5,85 & 8,93 & 4,00 & 1,67 \\
\hline Porque mi pareja ya tiene hijos & & 0,42 & 0,20 & 0,52 \\
\hline \multicolumn{5}{|l|}{ Motivos materiales } \\
\hline Carencia o carestía de escuelas infantiles & 0,01 & 0,15 & 0,10 & 0,09 \\
\hline Circunstancias de la vivienda/Malas condiciones de la vivienda & 0,05 & 0,75 & 0,90 & 0,56 \\
\hline Dificultad para conciliar la vidal laboral y familiar & 1,16 & 18,06 & 24,04 & 25,60 \\
\hline Entraría en conflicto con mi carrera profesional & 0,82 & 1,04 & 1,15 & 1,04 \\
\hline Exceso de trabajo en el hogar & 0,09 & 0,30 & 0,80 & 0,87 \\
\hline Insuficiencia de recursos económicos & 3,26 & 12,6 & 22,41 & 20,85 \\
\hline Por la situación laboral (propia o de la pareja) & 2,19 & 3,94 & 3,91 & 3,04 \\
\hline \multicolumn{5}{|l|}{ Motivos de salud } \\
\hline Demasiada edad para tener (más) hijos & 0,62 & 6,51 & 8,54 & 8,25 \\
\hline $\begin{array}{l}\text { No me quedaba embarazada/Dificultad para llevar un embarazo } \\
\text { a término }\end{array}$ & 2,29 & 11,97 & 6,61 & 5,65 \\
\hline Problemas o molestias de salud & 0,86 & 7,00 & 7,73 & 6,12 \\
\hline Dificultad para adoptar un/otro hijo & & 0,68 & 0,42 & 0,35 \\
\hline \multicolumn{5}{|l|}{ Otros motivos } \\
\hline No me gusta el modelo de sociedad actual para un niño & 0,54 & & & \\
\hline Preocupaciones/problemas que entraña criar a los hijos & 0,61 & 0,96 & 1,86 & 1,65 \\
\hline Temor a que el hijo/a nazca con problemas de salud & 0,25 & 1,18 & 1,20 & 1,21 \\
\hline Otros motivos* & 0,27 & 0,24 & 3,61 & 10,30 \\
\hline Total & $100 \%$ & $100 \%$ & $100 \%$ & $100 \%$ \\
\hline
\end{tabular}

*Nota: para mujeres a partir paridad 1, incluye aquellas que han tenido más hijos de los deseados (por ej.; partos múltiples), aunque después fueron excluidas del análisis.

Fuente: Encuesta de Fecundidad 2018, Instituto Nacional de Estadística 


\section{Apéndice 2 Método de cálculo de la fecundidad no realizada}

El método de cálculo de la fecundidad no realizada descompone la diferencia entre la fecundidad observada y la fecundidad deseada según sea el factor o razón principal que ha impedido alcanzar el número de hijos deseados hasta la fecha. El método solo tiene sentido cuando la fecundidad es inferior a la deseada. La versión que presentamos aquí está pensada para datos transversales. Hemos asumido tres supuestos:

1) Las edades representadas en la encuesta forman una cohorte sintética.

2) Los motivos que impiden alcanzar la fecundidad deseada son independientes entre ellos.

3) El efecto de eliminar un motivo a una determinad edad y paridad tiene efectos acumulativos a edades y paridades más altas.

Partiendo del primer supuesto, estimamos el Índice Sintético de Fecundidad (ISF) a partir de la fecundidad completa observada de las personas de más edad, en nuestro caso hemos tomado como referencia la edad 48 para mujeres y hombres.

A continuación, detallamos el método elaborado para el cálculo de la fecundidad no realizada paso a paso:

Paso 1: Eliminar el motivo que ha impedido alcanzar la fecundidad deseada hasta ese momento.

La proporción de mujeres que ha tenido un hijo a cierta edad se expresa como:

$$
P(x, i)=1-\sum_{h=1}^{i} \sum_{j=1}^{n} o_{j}(x, h)
$$

donde $x, i$ y $j$ representan la edad, paridad y el motivo, respectivamente. $n$ es el valor máximo de ese motivo. Nótese que $h$ también indica la paridad y $\{h \in Z: 1 \leq h \leq i\} .0_{j}(x, h)$ es la proporción de mujeres que no han tenido el hijo número $h$ debido al motivo $j$.

Siguiendo el segundo supuesto, la proporción hipotética de mujeres que hubiera tenido un primer hijo después de eliminar el motivo $j$ se define como:

$P_{j}^{*}(x, i)=P(x, i)+O_{j}(x, i)=1-\left[\sum_{h=1}^{i} \sum_{j=1}^{n} o_{j}(x, h)-o_{j}(x, i)\right]$.

Basándonos en esta ecuación (1), la fecundidad hipótetica resultado de eliminar el motivo $j$, $I S F_{j}$, se estima de la siguiente manera:

$\operatorname{TFR}_{j}^{*}(i)=P_{j}^{*}(X, i)$ 
donde $X$ es la edad máxima.

Paso 2. Cuantificar el efecto acumulativo de eliminar un motivo

Siguiendo los supuestos (2) y (3), el hecho de suprimir un motivo dentro de una paridad específica tiene un impacto sobre la distribución del resto de motivos en esa paridad y también en el número de nacimientos en paridades superiores. Para estimar este efecto acumulativo, simulamos un ISF hipotético para las paridades superiores resultado de suprimir un determinado motivo en paridades más bajas. Específicamente, distribuimos la proporción de mujeres que hubieran tenido el (i+1) hijo debido a la eliminación del motivo $j$, basándonos en la tasa de transición desde el hijo $i$ hasta el $(i+1)$ hijo.

La proporción de mujeres que hubiera tenido el hijo $(i+1)$ se calcula de la siguiente manera:

$$
\begin{array}{ll}
\pi_{j}(x, i)=P_{j}^{*}(x, i)-P_{j}^{*}(x-1, i), & x>\text { edad inicial } \\
\pi_{j}(x, i)=P_{j}^{*}(x, i), & x=\text { edad inicial }
\end{array}
$$

La tasa de transición a la edad $x$ se define como:

$$
\begin{array}{ll}
{ }_{i+1} \operatorname{TR}_{i}(x)=\frac{P(x, i+1)-P(x-1, i+1)}{P(x-1, i)-P(x-1, i+1)}, & x>\text { edad inicial } \\
{ }_{i+1} \operatorname{TR}_{i}(x)=0, & x=\text { edad inicial }
\end{array}
$$

Nótese que cuando la tasa de transición es negativa o mayor a 1, forzamos que estas tasas sean 0 o 1 respectivamente. Estas tasas inverosímiles pueden surgir debido a la naturaleza transversal de los datos.

El ISF hipotético de los nacimientos $(i+1)$ se define como:

$$
\operatorname{TFR}_{j}^{*}(i+1)=\sum_{x=a}^{X} f^{*}(x, i+1),
$$

donde $I(x, i+1)$ es la contribución a la hipotética tasa de fecundidad específica por edad a la edad $x$, que se calcula de la siguiente manera:

$$
f^{*}(x, i+1)=\sum_{\theta=1}^{X-x} I(x+\theta, i+1), \quad x \neq X-1
$$

donde $f^{*}(x, i+1)$ es la contribución de la hipotética tasa de fecundidad por edad del nacimiento $(i+1)$ a la edad $x$. $(X-x)$ es el rango entre la edad máxima y la edad específica, $x$. 


$$
\begin{array}{ll}
I(x+\theta, i+1)=\left[\pi_{j}(x, i)-\sum_{m=1}^{\theta-1} I(x+m, i+1)\right] i+1 R_{i}(x+\theta), & \theta \neq 1 \\
I(x+1, i+1)=\pi_{j}(x, i)_{i+1} T_{i}(x+1) . & \theta=1
\end{array}
$$

donde $\alpha$ es la edad inicial. $m$ y $\theta$ son la edad incremental.

Para poder trabajar con el grupo de edad abierto (o, cuando $x=X-1$ ),

$$
f^{*}(X-1, i+1)=I(X, i+1)=\left[\pi_{j}(X-1, i)+\pi_{j}(X, i)\right]_{i+1} T R_{i}(x+1) .
$$

Para calcular el término de iteración, $I(x+\theta, i+1)$, en la ecuación (6), sugerimos que las ecuaciones (5), (6) y (7) deberían ser calculada como un proceso completo en lugar de fórmulas separadas. Por ejemplo, si $\alpha=18, x=18$ y $=18$, y $i=1$ estas ecuaciones se reducirán a:

$$
\begin{aligned}
& T F R_{j}^{*}(2)=\sum_{x=18}^{48} f^{*}(x, 2) \\
& f^{*}(18,2)=\sum_{\theta=1}^{30} I(18+\theta, 2), \text { and } \\
& I(18+\theta, 2)=\left[\pi_{j}(18,1)-\sum_{m=1}^{\theta-1} I(18+m, 2)\right]{ }_{2}^{1} T R_{1}(18+\theta), \quad \theta \neq 1 \\
& I(19,2)=\pi_{j}(18,1) \frac{1}{2} T R_{1}(19) . \quad \theta=1
\end{aligned}
$$

En nuestro análisis, hemos trabajado con tres motivos principales que impiden alcanzar el número deseado de hijos: motivos de pareja, obstáculos materiales y problemas de salud. La suma de estos tres motivos es el efecto total de eliminar todos los motivos considerados. No hemos considerado como obstáculos que impiden alcanzar la fecundidad deseada los motivos relacionados "soy demasiado joven para tener un hijo/no estoy lista" y "no quiero tener hijos", puesto que creemos que reflejan las intenciones de no querer tener (más) hijos. 